\title{
3D Simulation of the Micro Indentation Process and Relative Problems Research
}

\section{Min Li Ling-Dong Wang, Nai-Gang Liang}

State Key Lab. of Nonlinear Mechanics, Institute of Mechanics(LNM), CAS, Beijing, 100080, China,Email:limin@lnm.imech.ac.cn

\begin{abstract}
In this paper the finite element method was used to simulate micro-scale indentation process. The several standard indenters were simulated with 3D finite element model. The emphasis of this paper was the differences between 2D axisymmetric cone model and 3D micro-scale indenter model. At last, the quantitative relationship between Vicker microhardness and nanoindentation hardness was given.
\end{abstract}

Keywords: microhardness nanoindentation finite element simulation

\section{Introduction}

The hardness test was a simple and effective method for evaluating the mechanical properties of material. For nearly one hundred years it was used widely in industry[1]. Recent years have seen significant improvements in indentation equipment and a growing need for measuring the mechanical properties of materials on small scales.

Nanoindentation technique has been presented and developed by Oliver[2]. It could provide load-displacement data of entire loading and unloading process. Compared with microhardness only providing hardness data, the nanoindentation technique give plenty and precise information that could be used to look for entire material properties.

As a main method, finite element method simulating micro-scale indentation process played important role in discussing how to get more and exact mechanical properties of material layer and better understanding experiment phenomenon. As the limitations of computer in speed and capability, Bhattacharya[3], who firstly used finite element method simulating micro-scale indentation process of homogeneous material, used the 2D axisymmetric cone model simulating Vicker indenter and Berkovich indenter.

In fact, the micro-scale indenters are not axisymmetric, and all material is uneven in micro-scale. These properties could not be presented in $2 \mathrm{D}$ cone model. In this paper the 3D model was used to simulate loading and unloading process with glide contact. The relationships between $2 \mathrm{D}$ and $3 \mathrm{D}$ simulation, microhardness and nanoindentation hardness were discussed.

\section{The 3D finite element model for micro- scale indentation process}

Berkovick indenter, the standard indenter of nanoindentation, was considered as object for finite element analysis. The geometric shape is illustrated as Fig. 1, and the 1/6 finite element model(short as FEM) according to geometric symmetry as Fig. 2 .

For achieving the calculating precision, mesh scale of FEM was made according to embedding depth. For nearly $1 \mu \mathrm{m}$ embedding 


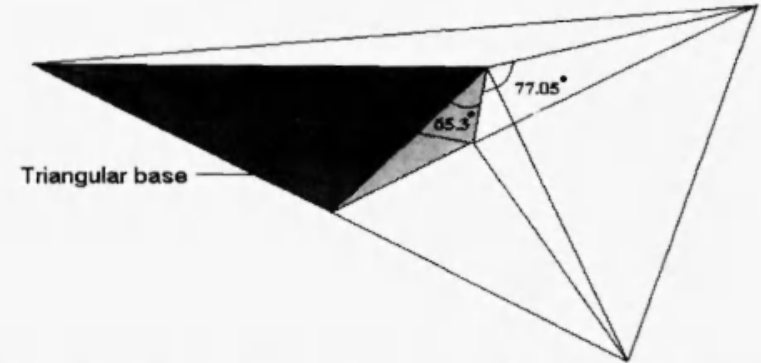

Fig.1. Shape and parameters of Berkovich indenter

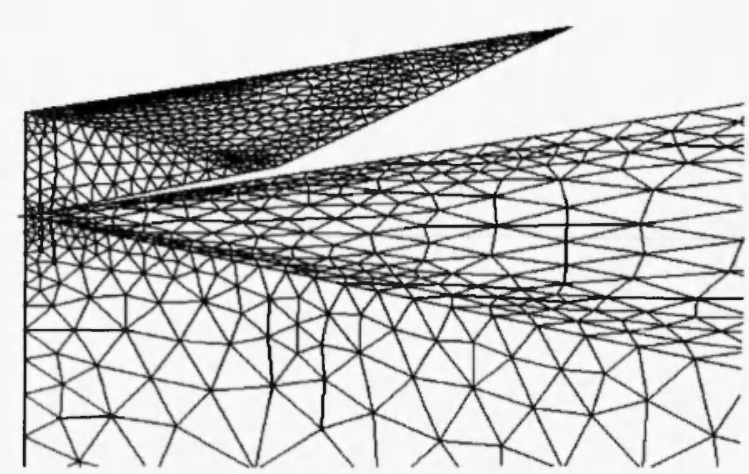

Fig. 2. A typical mesh for finite element model depth, the mesh scale of the contact region was about $50 \mathrm{~nm}$ which guarantees the calculating precision of more than $200 \mathrm{~nm}$ embedding depth. The four-node solid element was adopted for avoiding Jacobi matrix degenerating as material nonlinear.

Berkovich indenter made by diamond was modeled as elastic three-sided pyramid which Young's modulus and Poisson's ratio are $1141 \mathrm{GPa}$ and 0.07 , respectively. The specimen was modeled as a von Mises solid with discrete yielding followed by linear, isotropic work hardening. The input data was the uniaxial stress -strain curve.

The nonlinear quasi-static calculation process was realized on MSCNNASTRAN. Loading could be achieved by means of displace -ment or load control of the indenter. The loaddisplacement curves from the two methods were equivalent completely.

The response between sample and indenter was assumed frictionless, because calculations including friction(with coefficients of friction up to 1.0 ) showed no significant effect on results. The specimen dimensions was large enough to approximate the behavior of a semi-infinite halfspace, as evidenced by an insensitivity of results to further increases in specimen size.
For two materials including softer metal copper and harder metal tungsten, the nano indentation experiments were made to examine calculative results. The ratio of Young's modulus to yield $\operatorname{stress}\left(E / \sigma_{v}\right)$ of the two materials were 95.25 and 1280 which covered the region of major metal material. For avoiding calculative error and experimental error at the depth less than $100 \mathrm{~nm}$, the maximum depth of calculation and experiment was chosen to about $1 \mu \mathrm{m}$, and the corresponding loads were $23 \mathrm{mN}$ and $120 \mathrm{mN}$ for copper and tungsten, respective ly. The output of the finite element analysis included load-displacement curves during one cycle of loading and unloading, the shapes of contact impressions, and the geometries of plastic zones. The load-displacement results of calculation and experiment were shown in Fig.3.

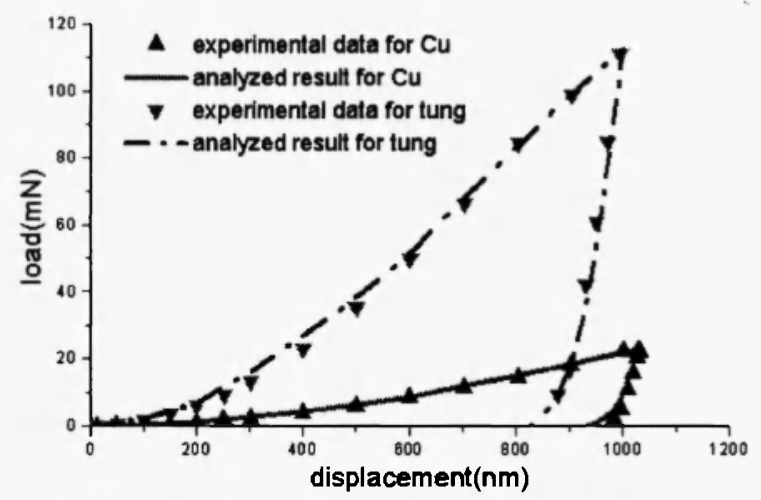

Fig.3. The results of experiment and calculation

\section{The relationship between $2 D$ and $3 D$ FEM simulation}

Though 3D FEM simulation could present the characteristics of indentation process with uneven material or non-axisymmetric indenter, the scale of $3 \mathrm{D}$ FEM was more than that of $2 \mathrm{D}$ cone FEM. If the aim of simulation was to get the rules in indentation process rather than numerical value, the $2 \mathrm{D}$ simulation was better. Using 2D cone FEM simulating 3D indenter with non-axisymmetric introduced an important problem: How to determine the angle on the tip of cone. Considering the characteristics of Vicker indenter, Bhattacharya[3] used directly a rigid cone with a semi-vertical angle $\phi=68^{\circ}$. 
Subsequently Sun[4] gave $\phi=70.3^{\circ}$ which gives the same volume-to-depth(projected areato-depth) ratio as Berkovich and Vicker indenter. In addition, some people used the rule of the same contact area-to-depth ratio.

At present, the standard indenter for microhardness included Vicker and Knoop indenter, and Berkovich indenter for nanoindentation. Aimed at the three kinds of indenters above, different cones(shown as Tab.1) according to different equivalent rule were used to simulate micro-scale indentation process. By means of comparing load-displacement curves and stress or strain field between $3 D$ indenter FEM and corresponding 2D cone FEM, the best rule was confirmed for increasing calculation efficiency based on kept veracity.

Table 1 the equivalent semi-vertical angles of several

\begin{tabular}{cccc}
\multicolumn{4}{c}{ type indenters } \\
\hline \multirow{2}{*}{ indenter } & $\begin{array}{c}\text { volume } \\
\text { equivalent }\end{array}$ & $\begin{array}{c}\text { contact area } \\
\text { equivalent }\end{array}$ & $\begin{array}{c}\text { Experi- } \\
\text { ential }\end{array}$ \\
\hline Berkovich & $70.32^{\circ}$ & $70.66^{\circ}$ & $68^{\circ}$ \\
Vicker & $70.30^{\circ}$ & $70.45^{\circ}$ & $68^{\circ}$ \\
Knoop & $77.64^{\circ}$ & $78.10^{\circ}$ & \\
\hline
\end{tabular}

Figure 4 gave plastic strain field of four indenters. The sample material was copper. The left and right sides of the indenter in figure presented two kinds of symmetrical faces.

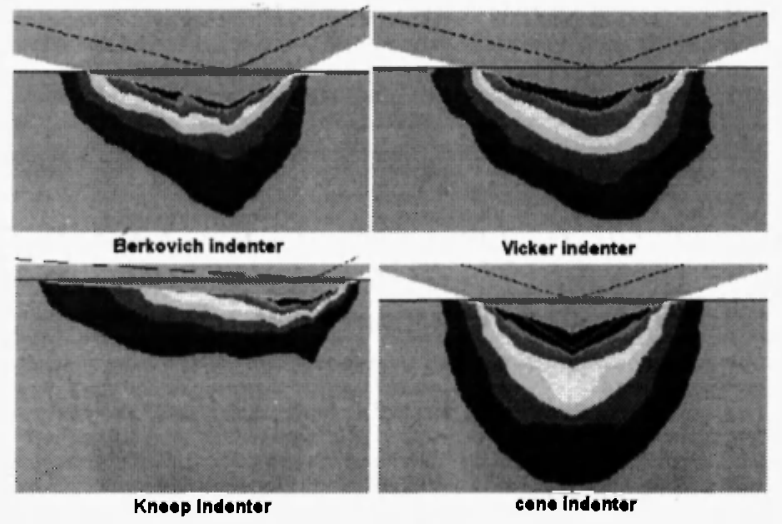

Fig.4. Plastic strain field of four indenters

With comparing the results in figures, the strain fields differed each other. The 2D simulation couldn't present the detail of the 3D simulation. Correspondingly, the strain fields of Vicker and Berkovich were similar to that of cone indenter, but the result of the knoop indenter differed completely with that of cone indenter. On the whole, it could be forecasted that the effects of cone simulation would be same sequence as above. This prediction was confirmed by load-displacement curves of different indenters as shown in Fig.5 to Fig.7.

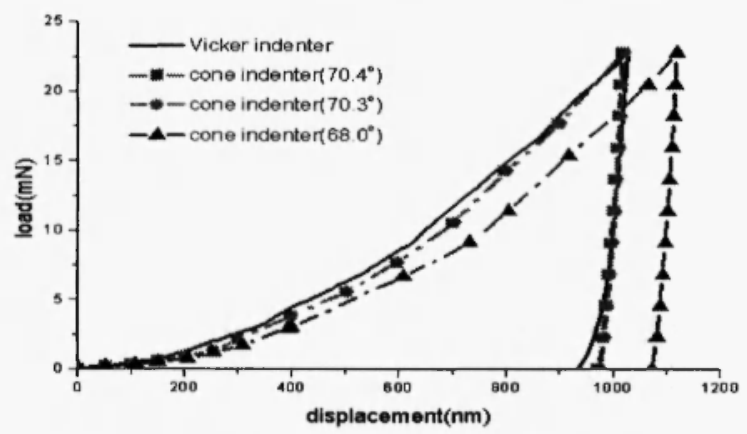

Fig.5. The results compared about Berkovich indenter

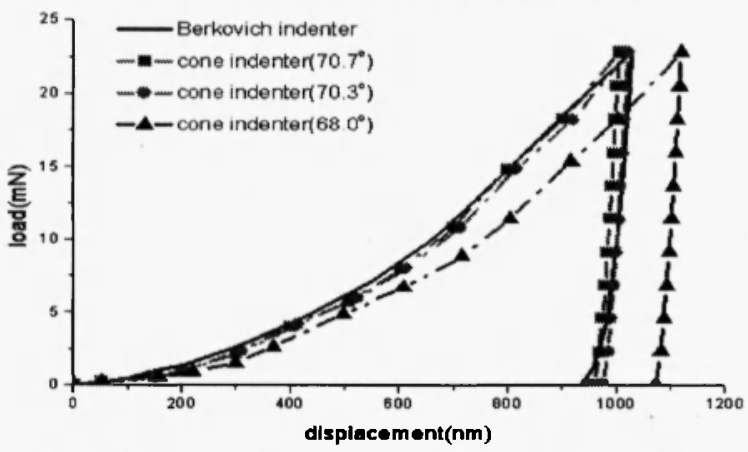

Fig.6. The results compared about Vicker indenter

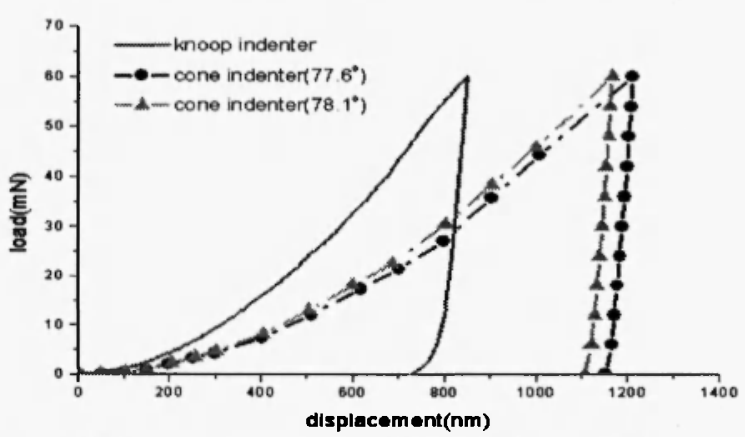

Fig.7. The results compared about Knoop indenter

For the regular pyramid indenters as Vicker and Berkovich, both volume equivalent and contact area equivalent rules could give good results, and the first one was better. The error of 2D simulation with $68^{\circ}$ semi-vertical angle was less $10 \%$, and this model could be accepted for discussing disciplinarians in indentation process. For Knoop indenter with unequal angles, both volume and contact area equivalent rules couldn't give right results, and the material 
hardness from 2D simulation would be overestimated. The conclusion was fit to the experiment data by Riester[5]. Via repetitiously calculating, the cone indenter with $81.7^{\circ}$ semivertical angle could give almost same loaddisplacement curve of Knoop indenter for metal copper, but for metal tungsten the angle changed to $79.4^{\circ}$. This was different from 2D simulation of Berkovich and Vicker which results were insensitive to material varying. It was the important factor that the ratio of the long diagonal to the short diagonal is too much.

\section{The relationship between microhard - ness and nanoindentation hardness}

At present, Vicker microhardness(short as VMH) and nanoindentation hardness(short as NH) were popular methods in micro-scale experiment. The load and impression area in the definition of $\mathrm{NH}$ were isochronous, but not true in microhardness which impression area was completely unloading area. In physics essence and experiment technique, microhardness differed in evidence from NH. Firstly, the difference was whether the elastic deformation was considered. Secondly, the impression area in $\mathrm{NH}$ was derived from load-displacement data, but in microhardness by imaging.

It was interest to compare Fig.5 and Fig.6 that the load-displacement curves of Berkovich and Vicker indenter were almost the same. So in theory, The VMH could be obtained by using completely unloading depth in load-displace ment curve of $\mathrm{NH}$. According to analyses above, the quantitative relationship between $\mathrm{NH}$ and VMH was shown in Fig.8.

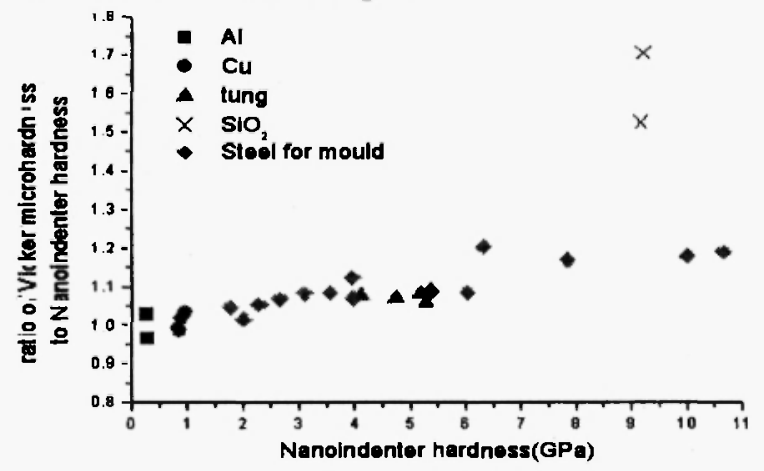

Fig.8. The dependence of $\mathrm{VMH} / \mathrm{NH}$ on $\mathrm{NH}$
The difference between $\mathrm{NH}$ and VMH was decided by the ratio of elastic deformation to total deformation. For small impression depth or the material with small value of $E / \sigma_{v}$, the elastic deformation could not be neglected so the quantitative difference between $\mathrm{NH}$ and VMH was evident such as $\mathrm{SiO}_{2}$. For metal material, the ratio increased slowly along with material hardness increasing, and the difference between them was less than $20 \%$.

\section{Conclusion}

1. For Vicker and Berkovich indenter, both volume equivalent and contact area equivalent rules would give good results, but the rules was not suitable for Knoop indenter.

2. The difference in physics essence between nanoindentation hardness and microhard ness was whether the elastic deformation was considered

\section{Acknowledgments}

The work is supported by Chinese Academy of Sciences Foundation(KGCXI-11), Post Doctor Science Foundation of China and K.C. Wong Education Foundation, Hong Kong..

\section{References}

[1] Tabor, D., Philo. Mag. A, Vol.74, No.5, 1996, pp 1207 1212

[2] Oliver, W.C, and Pharr, G.M, J. Mater. Res. Vol.7, No.6, 1992, pp1546 1583

[3] Bhattachary, A.K, and Nix, W.D, Int. J. of Solids and Structure, Vol.24, No.9, 1988, pp881-891

[4] Sun, Y, Bell, T, and Zheng, S, Thin Solid Films, Vol.258, 1995, pp 198 204

[5] Riester, L, Bell, T.J, Fischer-Cripps, A.C, J. Mater. Res., Vol.16, No.6, 2001, ppl6601667 